\title{
Intrinsic spin decay length in an antiferromagnetic insulator
}

\author{
Hiroto Sakimura, ${ }^{1,2}$ Akio Asami, ${ }^{2}$ Hiroki Hayashi, ${ }^{2}$ Takashi Harumoto, ${ }^{1}$ Yoshio Nakamura, ${ }^{1}$ Ji Shi, ${ }^{1}$ and Kazuya Ando ${ }^{2,3, *}$ \\ ${ }^{1}$ School of Materials and Chemical Technology, Tokyo Institute of Technology, Tokyo 152-8552, Japan \\ ${ }^{2}$ Department of Applied Physics and Physico-Informatics, Keio University, Yokohama 223-8522, Japan \\ ${ }^{3}$ Center for Spintronics Research Network (CSRN), Keio University, Yokohama 223-8522, Japan
}

(Received 5 February 2019; revised manuscript received 1 August 2019; published 20 August 2019)

\begin{abstract}
We report intrinsic spin decay length of an antiferromagnetic insulator. We found that at an antiferromagnetic/ferromagnetic interface, a spin current generated by spin pumping is strongly suppressed by two-magnon scattering. By eliminating the two-magnon contribution, we discovered that the characteristic length of spin decay in polycrystalline $\mathrm{NiO}$ changes by two orders of magnitude through the paramagnetic to antiferromagnetic transition. The spin decay length in the antiferromagnetic state is around $100 \mathrm{~nm}$, which is an order of magnitude longer than that previously believed. These results provide a crucial piece of information for the fundamental understanding of the physics of spin transport.
\end{abstract}

DOI: 10.1103/PhysRevResearch.1.013013

\section{INTRODUCTION}

Spintronics relies on the transport of spins in condensed matter [1-3]. Spin transport has been investigated in a variety of materials, including metals, semiconductors, and insulators. In metals and semiconductors, spins are transported by the diffusion of conduction electrons [3]. In contrast, in magnetically ordered materials, spins can be transported even in the absence of conduction electrons; spins are carried by the elementary excitations of magnetic moments, magnons [4]. The magnonic spin current in insulators is of particular recent interest because this sets a new direction for experimental and theoretical studies of the physics of spin transport $[5,6]$.

Antiferromagnetic insulators is a new class of materials for spin transport [7-9]. This class of materials potentially entails a number of advantages as compared to ferromagnets; antiferromagnets are robust against external magnetic fields, produce no stray fields, and display ultrafast dynamics. Since the first observation of the transmission of spins through an antiferromagnetic insulator $\mathrm{NiO}$ [10-12], intense experimental and theoretical efforts have been invested in unraveling the physics of the spin transport in antiferromagnetic insulators [10-22]. Recent studies have shown that antiferromagnets allow long-distance spin transport [21,22]. However, the long spin decay length has been observed only in single crystals using nonlocal techniques; the spin decay length in amorphous or polycrystalline antiferromagnets is known to be limited to only a few nanometers [9].

In this paper, we demonstrate that the spin decay length of polycrystalline $\mathrm{NiO}$, a prototypical antiferromagnetic in-

*Corresponding author should be addressed: ando@appi.keio.ac.jp

Published by the American Physical Society under the terms of the Creative Commons Attribution 4.0 International license. Further distribution of this work must maintain attribution to the author(s) and the published article's title, journal citation, and DOI. sulator, is around $100 \mathrm{~nm}$, which is an order of magnitude longer than that previously believed. We found that the spin decay length in $\mathrm{NiO}$ with columnar structure changes by two orders of magnitude through the paramagnetic to antiferromagnetic transition. These are evidenced by revealing the intrinsic character of magnonic spin transport by eliminating a two-magnon contribution in the spin-transmission signal. This result illustrates an essential role of the magnetic order in spin transport and shows the potential of polycrystalline antiferromagnetic insulators for good spin-current conductors.

\section{METHODS}

To quantify the intrinsic spin decay length of polycrystalline $\mathrm{NiO}$, we prepared $\mathrm{Ni}_{81} \mathrm{Fe}_{19}(8) / \mathrm{NiO}\left(d_{\mathrm{NiO}}\right) / \mathrm{Pt}(5)$ trilayers on thermally oxidized $\mathrm{Si}$ substrates by $\mathrm{rf}$ magnetron sputtering at room temperature [see Fig. 1(a)]. The numbers in brackets represent the thickness of each layer in nm unit, where $d_{\mathrm{NiO}}=0$ to $10.5 \mathrm{~nm}$. Here, the $\mathrm{NiO}$ layer is polycrystalline, which is evidenced by the x-ray diffractometry [23]. The $\mathrm{Ni}_{81} \mathrm{Fe}_{19}$ layer, capped by 4 -nm-thick $\mathrm{SiO}_{2}$, is a $1 \times 1.5 \mathrm{~mm}^{2}$ rectangular shape. For the $\mathrm{Ni}_{81} \mathrm{Fe}_{19} / \mathrm{NiO} / \mathrm{Pt}$ trilayers, we measured the spin pumping by varying a magnetic field $\mathbf{H}$ applied at an angle of $\theta_{H}$ from the film normal at room temperature [see Fig. 1(a)]. The spin pumping from the $\mathrm{Ni}_{81} \mathrm{Fe}_{19}$ layer injects a spin current into the $\mathrm{NiO}$ layer [24]. The spin current reaching the Pt layer is converted into an electric voltage $V_{\text {ISHE }}$ through the inverse spin Hall effect (ISHE) in the Pt layer [25], and thus the spin-current decay in the $\mathrm{NiO}$ layer can be characterized by measuring the $d_{\mathrm{NiO}}$ dependence of $V_{\text {ISHE }}$. In Fig. 1(b), we show the $H$ dependence of the microwave absorption intensity $I(H)$ and voltage $V(H)$ signals for the $\mathrm{Ni}_{81} \mathrm{Fe}_{19} / \mathrm{NiO} / \mathrm{Pt}$ trilayers with $d_{\mathrm{NiO}}=0$ and $4.1 \mathrm{~nm}$ at $\theta_{H}=90^{\circ}$. For the measurement, the $\mathrm{Ni}_{81} \mathrm{Fe}_{19} / \mathrm{NiO} / \mathrm{Pt}$ trilayer was placed at the center of a $\mathrm{TE}_{011}$ cavity with the frequency of $f=9.43 \mathrm{GHz}$ and power of $P=200 \mathrm{~mW}$, and we measured dc electric voltage $V$ between 

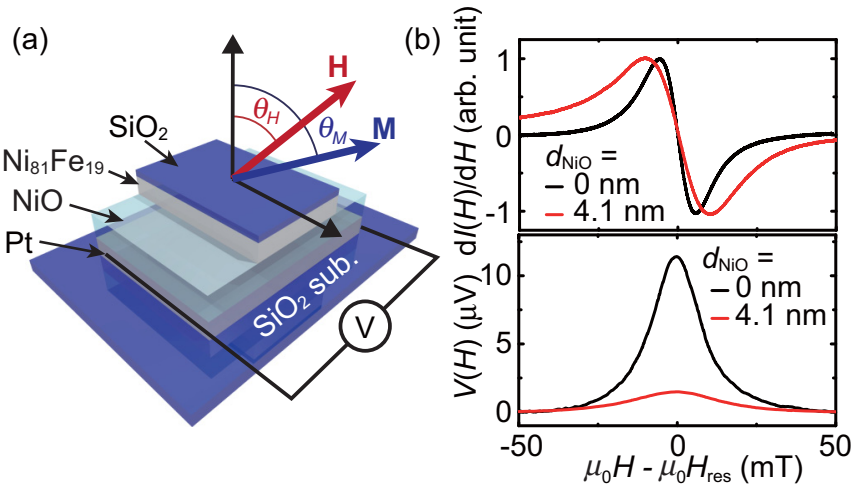

FIG. 1. (a) A schematic illustration of the $\mathrm{Ni}_{81} \mathrm{Fe}_{19} / \mathrm{NiO} / \mathrm{Pt}$ trilayer. $\mathbf{H}$ denotes the external magnetic field. $\mathbf{M}$ and $\theta_{M}$ represent the equilibrium direction of the magnetization when $\mathbf{H}$ is applied at an angle of $\theta_{H}$ from the film normal. (b) Magnetic field $H$ dependence of the microwave absorption signal $d I / d H$ and voltage signal $V$ for the $\mathrm{Ni}_{81} \mathrm{Fe}_{19} / \mathrm{NiO} / \mathrm{Pt}$ trilayers with $d_{\mathrm{NiO}}=0$ (black) and $4.1 \mathrm{~nm}$ (red). $I$ is the microwave absorption intensity and $H_{\text {res }}$ is the FMR field.

electrodes attached to the edges of the film [see Fig. 1(a)]. Figure 1(b) shows that the ISHE voltage $V_{\text {ISHE }}$ is generated around the FMR field $H=H_{\text {res. }}$. This result also shows that $V_{\text {ISHE }} \equiv V\left(H_{\text {res }}\right)$ is strongly suppressed by inserting the $\mathrm{NiO}$ layer, as expected for the spin-current decay in the antiferromagnet.

\section{RESULTS AND DISCUSSION}

Our finding is that magnetic-field angle $\theta_{H}$ dependence of $V_{\text {ISHE }}$ strongly depends on the $\mathrm{NiO}$ thickness $d_{\mathrm{NiO}}$. In Fig. 2(a), we show the $\theta_{H}$ dependence of $V_{\text {ISHE }}$ for the $\mathrm{Ni}_{81} \mathrm{Fe}_{19} / \mathrm{NiO} / \mathrm{Pt}$ trilayers with various $d_{\mathrm{NiO}}$. This result shows that the $\theta_{H}$ dependence of $V_{\mathrm{ISHE}}$ for the trilayers with different $d_{\mathrm{NiO}}$ is the same only around $\theta_{H}=0$. Here, the variation of $V_{\text {ISHE }}$ for the film with $d_{\mathrm{NiO}}=0 \mathrm{~nm}$ is consistent with the standard model of the spin pumping and ISHE [26]. In this model, when the magnetic damping constant $\alpha$ is independent of $\theta_{H}$, the spin current generated by the spin pumping is expressed as [26]

$$
j_{\mathrm{s}}\left(\theta_{M}\right)=\frac{g_{\mathrm{eff}}^{\uparrow \downarrow} h^{2} \hbar \gamma^{2} \omega}{4 \pi \alpha^{2} A\left(\theta_{M}\right)\left[\left(4 \pi M_{\mathrm{s}}\right)^{2} \gamma^{2} \sin ^{4} \theta_{M}+4 \omega^{2}\right]},
$$

where $g_{\text {eff }}^{\uparrow \downarrow}$ is the effective spin-mixing conductance, $h$ is the microwave magnetic field, $\gamma$ is the gyromagnetic ratio, $M_{\mathrm{s}}$ is the saturation magnetization, and $\omega=2 \pi f . \theta_{M}$ is the out-of-plane angle of the magnetization-precession axis [see Fig. 1(a)]. $A\left(\theta_{M}\right)=$ $2 \omega\left[4 \pi M_{\mathrm{s}} \gamma \sin ^{2} \theta_{M}+\sqrt{\left(4 \pi M_{\mathrm{s}} \gamma\right)^{2} \sin ^{4} \theta_{M}+4 \omega^{2}}\right]^{-1}$ is the precession ellipticity factor. When the magnetizationprecession axis is oblique to the film plane, the ISHE voltage $V_{\text {ISHE }}$ is proportional to $j_{\mathrm{s}}\left(\theta_{M}\right) \sin \theta_{M}$ because of $\mathbf{j}_{\mathrm{s}}^{\mathrm{Pt}} \| \mathbf{j}_{\mathrm{c}}^{\mathrm{Pt}} \times \boldsymbol{\sigma}$ [26], where $j_{\mathrm{s}}^{\mathrm{Pt}}$ is the spin current density injected into the Pt layer and $j_{\mathrm{c}}^{\mathrm{Pt}}$ is the charge current density generated by the ISHE. $\sigma$ is the spin-polarization direction of the spin current, which is parallel to the magnetization-precession axis. As shown in Fig. 2(a), this model well reproduces the experimental data only for $d_{\mathrm{NiO}}=0 \mathrm{~nm}$ (see the solid curve). For the calculation, we determined $\theta_{M}$ and $M_{\mathrm{s}}$ from measured $\theta_{H}$ dependence of $H_{\text {res }}$, shown in Fig. 2(b), by solving $\omega=\gamma \sqrt{H_{X} H_{Y}}$, where $\omega=2 \pi f, \quad H_{X}=H_{\text {res }} \cos \left(\theta_{H}-\theta_{M}\right)-M_{\text {eff }} \cos ^{2} \theta_{M}$, and $H_{Y}=H_{\text {res }} \cos \left(\theta_{H}-\theta_{M}\right)-M_{\text {eff }} \cos \left(2 \theta_{M}\right)$ [27-31] [see the inset to Figs. 2(b) and 2(c)]. $M_{\text {eff }} \simeq M_{\mathrm{s}}$ is the effective demagnetization field.

To clarify the origin of the anomaly in the $\theta_{H}$ dependence of $V_{\text {ISHE }}$ for the $\mathrm{Ni}_{81} \mathrm{Fe}_{19} / \mathrm{NiO} / \mathrm{Pt}$ trilayers with $d_{\mathrm{NiO}} \neq 0 \mathrm{~nm}$, we plot $\theta_{M}$ dependence of $V_{\text {ISHE }}$ in Fig. 2(c). Since $j_{\mathrm{c}}^{\mathrm{Pt}}\left(\theta_{M}\right)$ does not change drastically with $\theta_{M}, V_{\text {ISHE }}$ is approximately proportional to $\sin \theta_{M}$. In fact, the $\theta_{M}$ dependence of $V_{\text {ISHE }}$ is consistent with this scenario for the $\mathrm{Ni}_{81} \mathrm{Fe}_{19} / \mathrm{Pt}$ bilayer $\left(d_{\mathrm{NiO}}=0 \mathrm{~nm}\right)$. However, for the $\mathrm{Ni}_{81} \mathrm{Fe}_{19} / \mathrm{NiO} / \mathrm{Pt}$ trilayers, the measured $V_{\text {ISHE }}$ values are proportional to $\sin \theta_{M}$ only at $\left|\theta_{M}\right|<45^{\circ}$ as shown in Fig. 2(c); $V_{\text {ISHE }}$ deviates from $\sin \theta_{M}$ at $\left|\theta_{M}\right|>45^{\circ}$ with increasing the thickness of the $\mathrm{NiO}$ layer.

The drastic change in $V_{\text {ISHE }}$ at $\left|\theta_{M}\right|>45^{\circ}$ indicates that the nontrivial variation of $V_{\mathrm{ISHE}}$ is caused by two-magnon scattering in the $\mathrm{Ni}_{81} \mathrm{Fe}_{19} / \mathrm{NiO} / \mathrm{Pt}$ trilayers. The two-magnon scattering can be induced only when $\left|\theta_{M}\right|>45^{\circ}$ because the degenerated states with $k=0$ mode disappear at $\left|\theta_{M}\right|<45^{\circ}$
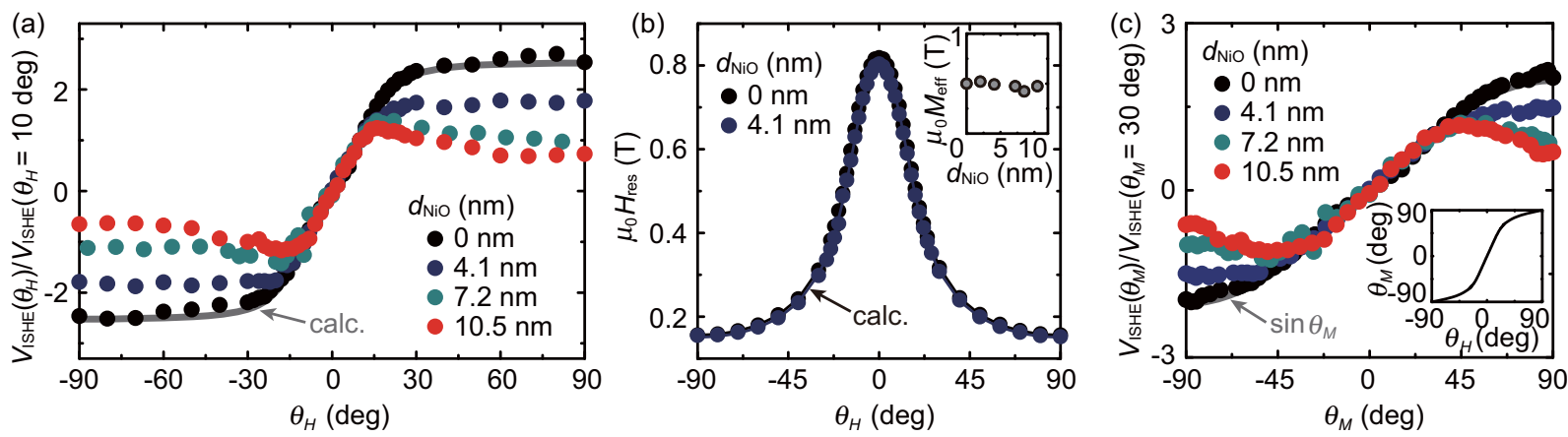

FIG. 2. (a) Out-of-plane magnetic field angle $\theta_{H}$ dependence of the ISHE voltage $V_{\mathrm{ISHE}}$ for the $\mathrm{Ni}_{81} \mathrm{Fe}_{19} / \mathrm{NiO} / \mathrm{Pt}$ trilayers with $d_{\mathrm{NiO}}=0$, 4.1, 7.2, and $10.5 \mathrm{~nm}$. The solid curve is the theoretical prediction based on the model of the spin pumping and ISHE, $j_{\mathrm{s}}\left(\theta_{M}\right) \sin \theta_{M}$. (b) $\theta_{H}$ dependence of the FMR field $H_{\mathrm{res}}$ for $d_{\mathrm{NiO}}=0$ and $4.1 \mathrm{~nm}$. The solid curves are the fitting results. The inset sows the $d_{\mathrm{NiO}}$ dependence of the effective demagnetization field $M_{\text {eff }}$. (c) Out-of-plane angle of the magnetization-precession axis, $\theta_{M}$, dependence of $V_{\text {ISHE }}$ for the $\mathrm{Ni}_{81} \mathrm{Fe}_{19} / \mathrm{NiO} / \mathrm{Pt}$ trilayers. The solid curve is a function proportional to $\sin \theta_{M}$. The inset shows $\theta_{H}$ dependence of $\theta_{M}$ for $d_{\mathrm{NiO}}=0$. 


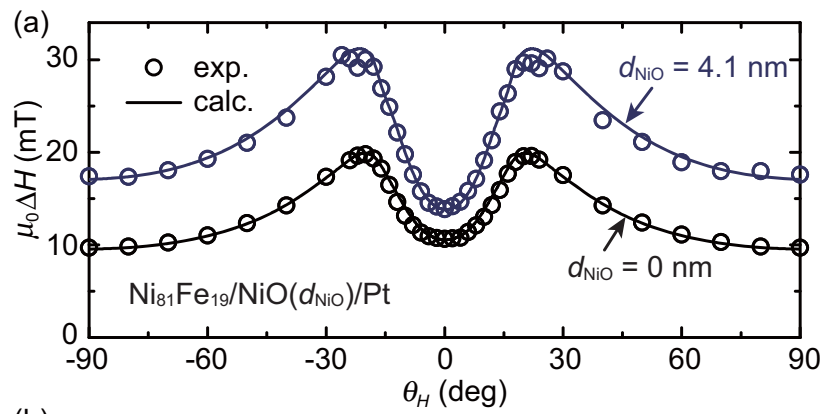

(b)
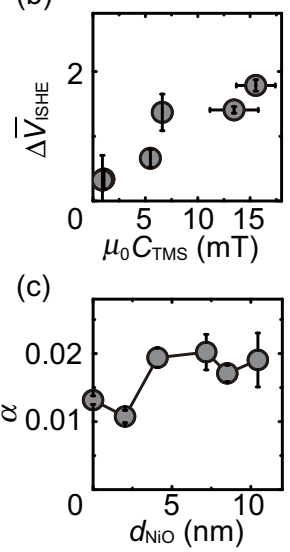

(d)

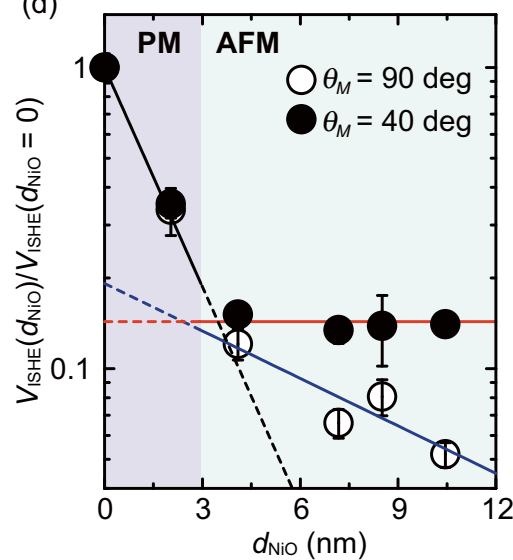

FIG. 3. (a) $\theta_{H}$ dependence of the peak-to-peak FMR linewidth $\Delta H$ for $d_{\mathrm{NiO}}=0 \mathrm{~nm}$ and $4.1 \mathrm{~nm}$. The open circles are the experimental data and the solid curves are the fitting results [23]. (b) The relation between the amplitude of the two-magnon scattering $C_{\mathrm{TMS}}$ and $\Delta \bar{V}_{\mathrm{ISHE}} \equiv V_{\mathrm{ISHE}}^{\mathrm{calc}}\left(\theta_{H}=90^{\circ}\right) / V_{\mathrm{ISHE}}^{\text {calc }}\left(\theta_{H}=10^{\circ}\right)-$ $V_{\text {ISHE }}^{\text {exp }}\left(\theta_{H}=90^{\circ}\right) / V_{\text {ISHE }}^{\text {exp }}\left(\theta_{H}=10^{\circ}\right)$, where $V_{\text {ISHE }}^{\text {calc }(\exp )}\left(\theta_{H}\right)$ is the calculated(measured) ISHE voltage at $\theta_{H}$. (c) $d_{\mathrm{NiO}}$ dependence of the magnetic damping constant $\alpha$. (d) $d_{\mathrm{NiO}}$ dependence of $V_{\mathrm{ISHE}}$ at $\theta_{M}=90^{\circ}$ (open circles) and $40^{\circ}$ (solid circles). The solid line in black is the fitting result using an exponential function, $\exp \left(-d_{\mathrm{NiO}} / \lambda_{\mathrm{NiO}}\right)$, for $d_{\mathrm{NiO}}<3 \mathrm{~nm}$. The solid lines in blue and red are the fitting result for the data at $\theta_{M}=90^{\circ}$ and $\theta_{M}=40^{\circ}$ for $d_{\mathrm{NiO}}>3 \mathrm{~nm}$, respectively.

[30-32]. Here, as shown in Fig. 1(b), the peak-to-peak FMR linewidth $\Delta H$ is clearly enhanced by inserting the $\mathrm{NiO}$ layer, despite the negligible change in the effective demagnetization field $M_{\text {eff }}$ [see the inset to Fig. 2(b)]. To quantitatively study the damping enhancement induced by the $\mathrm{NiO}$ insertion, we plot $\theta_{H}$ dependence of $\Delta H$ in Fig. 3(a). Figure 3(a) shows $\Delta H\left(\theta_{H}=\theta_{M}=0^{\circ}\right) \simeq \Delta H\left(\theta_{H}=\theta_{M}=90^{\circ}\right)$ for $d_{\mathrm{NiO}}=0$ $\mathrm{nm}$, while $\Delta H\left(\theta_{H}=\theta_{M}=0^{\circ}\right)<\Delta H\left(\theta_{H}=\theta_{M}=90^{\circ}\right)$ for $d_{\mathrm{NiO}}=4.1 \mathrm{~nm}$. This result indicates that $\Delta H\left(\theta_{H}=90^{\circ}\right)$ for the $\mathrm{Ni}_{81} \mathrm{Fe}_{19} / \mathrm{NiO} / \mathrm{Pt}$ trilayer is influenced by the two-magnon scattering. In fact, the measured $\theta_{H}$ dependence of $\Delta H$ is well reproduced by a calculation which takes into account the additional damping due to the two-magnon scattering as shown in Fig. 3(a) [28,33] (for details, see Ref. [23]). To clarify the relation between the amplitude of the two-magnon scattering $C_{\mathrm{TMS}}$ and the voltage suppression, we characterize the suppression of $V_{\text {ISHE }}$ induced by the $\mathrm{NiO}$ insertion as the difference between the measured $V_{\text {ISHE }}$ and $V_{\text {ISHE }}$ calculated using the conventional spin-pumping model, $\Delta \bar{V}_{\mathrm{ISHE}} \equiv V_{\mathrm{ISHE}}^{\text {calc }}\left(\theta_{H}=90^{\circ}\right) / V_{\mathrm{ISHE}}^{\text {calc }}\left(\theta_{H}=10^{\circ}\right)-V_{\mathrm{ISHE}}^{\text {exp }}\left(\theta_{H}=\right.$ $\left.90^{\circ}\right) / V_{\text {ISHE }}^{\text {exp }}\left(\theta_{H}=10^{\circ}\right)$, where $V_{\text {ISHE }}^{\text {calc }}\left(\theta_{H}\right)$ and $V_{\text {ISHE }}^{\text {exp }}\left(\theta_{H}\right)$ are the calculated and measured ISHE voltage at $\theta_{H}$, respectively [see Fig. 2(a)]. In Fig. 3(b), we plot $\Delta \bar{V}_{\text {ISHE }}$ with respect to $C_{\text {TMS }}$, extracted by the calculation shown in Fig. 3(a). As shown in Fig. 3(b), $\Delta \bar{V}_{\text {ISHE }}$ increases with $C_{\text {TMS }}$, supporting that the suppressed $V_{\text {ISHE }}$ signals at $\left|\theta_{M}\right|>45^{\circ}$ is caused by the two-magnon scattering.

The two-magnon scattering in the $\mathrm{Ni}_{81} \mathrm{Fe}_{19} / \mathrm{NiO} / \mathrm{Pt}$ trilayers is caused by a random fluctuation of the exchange bias, which is inevitable in ferromagnetic/antiferromagnetic junctions. The two-magnon scattering is known to be activated by the random fluctuation of uniaxial anisotropy, surface/interface roughness, and defects [28-31,34]. Here, we found that the surface roughness of the $\mathrm{NiO}$ layer is almost unchanged with $d_{\mathrm{NiO}}$ [23], showing that the two-magnon scattering cannot be attributed to the roughness effect because the two-magnon scattering increases with $d_{\mathrm{NiO}}$ in the $\mathrm{Ni}_{81} \mathrm{Fe}_{19} / \mathrm{NiO} / \mathrm{Pt}$ trilayer [see Fig. 2(c)]. We note that in the $\mathrm{Ni}_{81} \mathrm{Fe}_{19} / \mathrm{NiO} / \mathrm{Pt}$ trilayers, the $\mathrm{NiO}$ layer is polycrystalline, as evidenced by the $\mathrm{x}$-ray diffractometry [23]. This suggests that the two-magnon scattering is induced by the random fluctuation of uniaxial anisotropy due to randomly oriented exchange bias fields, which is caused by statistical distribution of antiferromagnetic grains in the polycrystalline $\mathrm{NiO}$ layer [33]. The random fluctuation of uniaxial anisotropy due to the randomly oriented exchange bias increases with $d_{\mathrm{NiO}}$ [33], and thus the amplitude of the two-magnon scattering $C_{\mathrm{TMS}}$ increases with $d_{\mathrm{NiO}}$, which is consistent with the enhancement of the two-magnon scattering by increasing $d_{\mathrm{NiO}}$ in the $\mathrm{Ni}_{81} \mathrm{Fe}_{19} / \mathrm{NiO} / \mathrm{Pt}$ device.

From the calculation of the $\theta_{H}$ dependence of $\Delta H$, we also extracted the damping constant $\alpha=\mu_{0}\left[\Delta H-\left(\Delta H_{\text {inhomo }}+\right.\right.$ $\left.\left.\Delta H_{\mathrm{TMS}}\right)\right](\sqrt{3} / 2)(\gamma \Xi / \omega)$, where $\Delta H_{\text {inhomo }}$ and $\Delta H_{\mathrm{TMS}}$ are the linewidth due to inhomogeneity and two-magnon scattering, respectively. $\Xi$ is the dragging function [23]. Figure 3(c) shows that $\alpha$ decreases at $d_{\mathrm{NiO}}=2.0 \mathrm{~nm}$, while $\alpha$ increases above $d_{\mathrm{NiO}}=4.1 \mathrm{~nm}$, consistent with previous reports [10,12]; $\alpha$ decreases due to the decoupling of the $\mathrm{Ni}_{81} \mathrm{Fe}_{19}$ and Pt layers by the insulating and nonNéel-ordered $\mathrm{NiO}$ layer because the Néel temperature of 2-nm-thick $\mathrm{NiO}$ is below the room temperature [35-37]. Above $d_{\mathrm{NiO}}=4.1 \mathrm{~nm}, \alpha$ increases because of the enhanced antiferromagnetic correlation due to the thickness growth $[10,38]$.

Commonly, the spin decay length $\lambda_{\mathrm{NiO}}$ of $\mathrm{NiO}$ is obtained from the thickness $d_{\mathrm{NiO}}$ dependence of $V_{\mathrm{ISHE}}$ at $\theta_{H}=\theta_{M}=$ $90^{\circ}$ [10-12]. Following this procedure, we plot the $d_{\mathrm{NiO}}$ dependence of $V_{\text {ISHE }}$ at $\theta_{M}=90^{\circ}$ in Fig. 3(d). This result shows that the spin decay length is increased from $\lambda_{\mathrm{NiO}}=1.9$ $\mathrm{nm}$ for $d_{\mathrm{NiO}}<3 \mathrm{~nm}$ to $\lambda_{\mathrm{NiO}}=7.9 \pm 2.3 \mathrm{~nm}$ for $d_{\mathrm{NiO}}>3 \mathrm{~nm}$. The increase of $\lambda_{\mathrm{NiO}}$ can be attributed to the paramagnetic to antiferromagnetic transition; for $d_{\mathrm{NiO}}<3 \mathrm{~nm}$, the Néel temperature is lower than the room temperature, while the $\mathrm{NiO}$ layer with $d_{\mathrm{NiO}}>3 \mathrm{~nm}$ is antiferromagnetic at room temperature $[10,35,36] . \lambda_{\mathrm{NiO}} \simeq 8 \mathrm{~nm}$ in the antiferromagnetic state is consistent with previous reports [10,12]. However, we note that, as is clear from Fig. 2(a), the $V_{\text {ISHE }}$ signals at $\theta_{H}=$ $90^{\circ}$ are strongly suppressed by the two-magnon scattering. This results in underestimation of the spin decay length in the antiferromagnetic state because the voltage suppression increases with $d_{\mathrm{NiO}}$. 


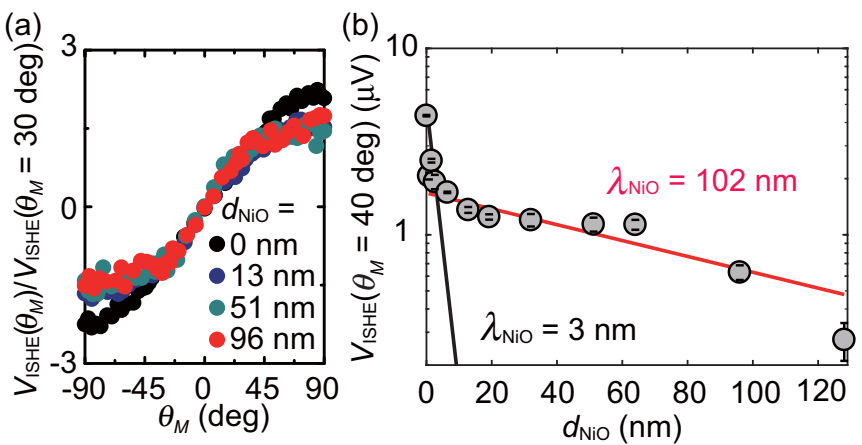

FIG. 4. (a) $\theta_{M}$ dependence of $V_{\text {ISHE }}$ for the $\mathrm{Ni}_{81} \mathrm{Fe}_{19} / \mathrm{NiO} / \mathrm{Pt}$ trilayers with $d_{\mathrm{NiO}}=0,13,51$, and $96 \mathrm{~nm}$. (b) $d_{\mathrm{NiO}}$ dependence of $V_{\text {ISHE }}$ at $\theta_{M}=40^{\circ}$. The solid line in black is the fitting result using an exponential function, $\exp \left(-d_{\mathrm{NiO}} / \lambda_{\mathrm{NiO}}\right)$, for $d_{\mathrm{NiO}}<2 \mathrm{~nm}$. The solid line in red is the fitting result for $d_{\mathrm{NiO}}>6 \mathrm{~nm}$.

The intrinsic spin decay length, where the two-magnon contribution is excluded, can be determined only from the $d_{\mathrm{NiO}}$ dependence of $V_{\text {ISHE }}$ at $\left|\theta_{M}\right|<45^{\circ}$, where the voltage suppression due to the two-magnon scattering is absent. As shown in Fig. 3(d), the $d_{\mathrm{NiO}}$ dependence of $V_{\mathrm{ISHE}}$ at $\theta_{M}=40^{\circ}$ is clearly different from that at $\theta_{M}=90^{\circ}$; at $\theta_{M}=40^{\circ}, V_{\mathrm{ISHE}}$ is almost independent of $d_{\mathrm{NiO}}$, indicating that $\lambda_{\mathrm{NiO}}$ is much larger than $10 \mathrm{~nm}$.

To quantify the intrinsic spin decay length in $\mathrm{NiO}$, we measured the $\theta_{H}$ dependence of $V_{\text {ISHE }}$ for $\mathrm{Ni}_{81} \mathrm{Fe}_{19} / \mathrm{NiO} / \mathrm{Pt}$ devices with large $d_{\mathrm{NiO}}$, as shown in Fig. 4(a). The devices are a different batch from those used to obtain the data shown in Figs. 1-3. The NiO layer is composed of dense columns with in-plane size of around $20 \mathrm{~nm}$ as confirmed by transmission electron microscopy (TEM) and atomic force microscopy [23]. Figure 4(a) shows that $V_{\text {ISHE }}$ is suppressed by the twomagnon scattering, although the two-magnon effect is weaker than that in Fig. 2(c). This result also shows that $V_{\text {ISHE }}$ can be observed even for $d_{\mathrm{NiO}}=128 \mathrm{~nm}$. We have checked the insulating property of the $\mathrm{NiO}$ layer and found that the resistance of the $\mathrm{NiO}$ layer is high enough to electrically separate the $\mathrm{Pt}$ and $\mathrm{Ni}_{81} \mathrm{Fe}_{19}$ layers [23]. Thus the measured signals can only be attributed to the ISHE voltage generated in the Pt layer.

In Fig. 4(b), we plot the $d_{\mathrm{NiO}}$ dependence of $V_{\mathrm{ISHE}}$ at $\theta_{M}=$ $40^{\circ}$. This result shows that the intrinsic spin decay length of the polycrystalline $\mathrm{NiO}$ layer in the antiferromagnetic state is $\lambda_{\mathrm{NiO}}=102 \pm 32 \mathrm{~nm}$, which is almost ten times longer than previously reported values $[10,12]$. This result shows that the characteristic length of spin decay in $\mathrm{NiO}$ changes by two-orders of magnitude through the paramagnetic to antiferromagnetic transition, illustrating the crucial role of the antiferromagnetic order for efficient spin transport in antiferromagnetic insulators.

\section{CONCLUSIONS}

In summary, we investigated magnonic spin transport in an antiferromagnetic insulator $\mathrm{NiO}$. We found that in the in-plane magnetic field geometry, the spin transport signal is strongly suppressed by the two-magnon scattering. Our result shows that the intrinsic spin decay length of polycrystalline $\mathrm{NiO}$ with columnar structure is around $100 \mathrm{~nm}$, which is an order of magnitude longer than that previously believed. We note that the two-magnon scattering at the $\mathrm{Ni}_{81} \mathrm{Fe}_{19} / \mathrm{NiO}$ interface arises from the randomly oriented exchange bias fields, which is inevitable in ferromagnet/antiferromagnet junctions. This indicates that the effect from the two-magnon scattering is ubiquitous in antiferromagnetic spin-transport studies via various techniques including the spin Seebeck and spin pumping. As long as a ferromagnet is used as a spin current injector, the two-magnon scattering masks the intrinsic spin transport properties in antiferromagnets.

\section{ACKNOWLEDGMENTS}

This work was supported by JSPS KAKENHI Grants No. 19H00864, No. 26220604, and No. 26103004, the Canon Foundation, the Asahi Glass Foundation, JGC-S Scholarship Foundation, and Spintronics Research Network of Japan (Spin-RNJ). H.S. is supported by JSPS Grant-in-Aid for Research Fellowship for Young Scientists (DC1) No. JP17J03624.
[1] I. Žutić, J. Fabian, and S. Das Sarma, Rev. Mod. Phys. 76, 323 (2004).

[2] Concepts in Spin Electronics, edited by S. Maekawa (Oxford University Press, Oxford, 2006).

[3] Spin Current, edited by S. Maekawa, S. O. Valenzuela, E. Saitoh, and T. Kimura (Oxford University Press, Oxford, 2012).

[4] Y. Kajiwara, K. Harii, S. Takahashi, J. Ohe, K. Uchida, M. Mizuguchi, H. Umezawa, H. Kawai, K. Ando, K. Takanashi, S. Maekawa, and E. Saitoh, Nature (London) 464, 262 (2010).

[5] L. Cornelissen, J. Liu, R. Duine, J. B. Youssef, and B. Van Wees, Nat. Phys. 11, 1022 (2015).

[6] D. Wesenberg, T. Liu, D. Balzar, M. Wu, and B. L. Zink, Nat. Phys. 13, 987 (2017).

[7] T. Jungwirth, X. Marti, P. Wadley, and J. Wunderlich, Nat. Nanotechnol. 11, 231 (2016).

[8] M. B. Jungfleisch, W. Zhang, and A. Hoffmann, Phys. Lett. A 382, 865 (2018).
[9] V. Baltz, A. Manchon, M. Tsoi, T. Moriyama, T. Ono, and Y. Tserkovnyak, Rev. Mod. Phys. 90, 015005 (2018).

[10] H. Wang, C. Du, P. C. Hammel, and F. Yang, Phys. Rev. Lett. 113, 097202 (2014).

[11] C. Hahn, G. De Loubens, V. V. Naletov, J. B. Youssef, O. Klein, and M. Viret, Europhys. Lett. 108, 57005 (2014).

[12] H. Wang, C. Du, P. C. Hammel, and F. Yang, Phys. Rev. B 91, 220410(R) (2015).

[13] T. Moriyama, S. Takei, M. Nagata, Y. Yoshimura, N. Matsuzaki, T. Terashima, Y. Tserkovnyak, and T. Ono, Appl. Phys. Lett. 106, 162406 (2015).

[14] S. Takei, T. Moriyama, T. Ono, and Y. Tserkovnyak, Phys. Rev. B 92, 020409(R) (2015).

[15] S. Seki, T. Ideue, M. Kubota, Y. Kozuka, R. Takagi, M. Nakamura, Y. Kaneko, M. Kawasaki, and Y. Tokura, Phys. Rev. Lett. 115, 266601 (2015). 
[16] W. Lin, K. Chen, S. Zhang, and C. L. Chien, Phys. Rev. Lett. 116, 186601 (2016).

[17] S. Takei, B. I. Halperin, A. Yacoby, and Y. Tserkovnyak, Phys. Rev. B 90, 094408 (2014).

[18] S. M. Rezende, R. L. Rodríguez-Suárez, and A. Azevedo, Phys. Rev. B 93, 054412 (2016).

[19] S. A. Bender, H. Skarsvåg, A. Brataas, and R. A. Duine, Phys. Rev. Lett. 119, 056804 (2017).

[20] A. Qaiumzadeh, H. Skarsvåg, C. Holmqvist, and A. Brataas, Phys. Rev. Lett. 118, 137201 (2017).

[21] R. Lebrun, A. Ross, S. Bender, A. Qaiumzadeh, L. Baldrati, J. Cramer, A. Brataas, R. Duine, and M. Kläui, Nature (London) 561, 222 (2018).

[22] W. Yuan, Q. Zhu, T. Su, Y. Yao, W. Xing, Y. Chen, Y. Ma, X. Lin, J. Shi, R. Shindou et al., Sci. Adv. 4, eaat1098 (2018).

[23] See Supplemental Material at http://link.aps.org/supplemental/ 10.1103/PhysRevResearch.1.013013 for a detailed description of sample fabrication and characterization and evaluation of two-magnon scattering amplitude.

[24] Y. Tserkovnyak, A. Brataas, and G. E. W. Bauer, Phys. Rev. B 66, 224403 (2002).

[25] E. Saitoh, M. Ueda, H. Miyajima, and G. Tatara, Appl. Phys. Lett. 88, 182509 (2006).
[26] K. Ando, S. Takahashi, J. Ieda, Y. Kajiwara, H. Nakayama, T. Yoshino, K. Harii, Y. Fujikawa, M. Matsuo, S. Maekawa, and E. Saitoh, J. Appl. Phys. 109, 103913 (2011).

[27] S. Mizukami, Y. Ando, and T. Miyazaki, Phys. Rev. B 66 , 104413 (2002).

[28] J. Lindner, I. Barsukov, C. Raeder, C. Hassel, O. Posth, R Meckenstock, P. Landeros, and D. L. Mills, Phys. Rev. B 80, 224421 (2009).

[29] P. Landeros, R. E. Arias, and D. L. Mills, Phys. Rev. B 77, 214405 (2008).

[30] R. Arias and D. L. Mills, Phys. Rev. B 60, 7395 (1999).

[31] R. Arias and D. Mills, J. Appl. Phys. 87, 5455 (2000).

[32] H. Kurebayashi, T. Skinner, K. Khazen, K. Olejník, D. Fang, C. Ciccarelli, R. Campion, B. Gallagher, L. Fleet, A. Hirohata et al., Appl. Phys. Lett. 102, 062415 (2013).

[33] H. Sakimura, A. Asami, T. Harumoto, Y. Nakamura, J. Shi, and K. Ando, Phys. Rev. B 98, 144406 (2018).

[34] A. Azevedo, A. B. Oliveira, F. M. De Aguiar, and S. M. Rezende, Phys. Rev. B 62, 5331 (2000).

[35] M. Gruyters, J. Magn. Magn. Mater. 248, 248 (2002).

[36] A. Baruth and S. Adenwalla, Phys. Rev. B 78, 174407 (2008).

[37] X. Wang, C. O. Pauyac, and A. Manchon, Phys. Rev. B 89, 054405 (2014).

[38] X. Lang, W. Zheng, and Q. Jiang, Nanotechnology 18, 155701 (2007). 